\title{
High-Resolution Ultrasonography in the Assessment of Lumbar Radiculoplexus Neuropathy in Diabetics: Preliminary Results of a Case-Control Study
}

\author{
Reddy Ravikanth ${ }^{1}$ (๑) \\ ${ }^{1}$ Department of Radiology, St. John's Hospital, Bengaluru, Karnataka, \\ Address for correspondence Reddy Ravikanth, MD, Department of \\ India \\ Radiology, St. John's Hospital, Bengaluru 560034, Karnataka, India \\ (e-mail: ravikanthreddy06@gmail.com). \\ J Neurosci Rural Pract 2021;12:792-795.
}

\begin{abstract}
\section{Keywords}

- lumbar radiculopathy

- ultrasonography

- anatomy

- cross-sectional area
\end{abstract}

Background The traditional diagnosis of lumbar radiculoplexus neuropathy (LRN) is based on a classical sequence of symptoms and targeted electrodiagnostic examination by means of electromyography. Ultrasonography reliably indicates the level of lumbar radiculopathy by assessing edema mesial to the site of compression.

Materials and Methods This case-control study was undertaken at a tertiary care hospital between July 2017 and June 2019 on 15 diabetic patients with symptoms of LRN. Fifteen healthy volunteers with no symptoms or clinical signs of LRN were included in the control group. The diameter (D) and transverse diameter (TD) of L1 nerve root (L1NR), L2NR, L3NR, and L4NR were measured, and their cross-sectional areas (CSAs) were calculated based on location in the lateral zone, where the NRs were visualized. On high-resolution ultrasonography, femoral nerve was localized lateral to the femoral artery in the femoral triangle beneath the inguinal ligament. Additionally, the CSA (calculated as CSA $\left.\left[\mathrm{mm}^{2}\right]=\mathrm{D} \times \mathrm{TD} \times \pi / 4\right)$, the diameter $(\mathrm{mm})$, and transverse diameter $(\mathrm{mm})$ of bilateral femoral nerves at the level of L3-L4 were calculated.

Results The difference attributed to CSAs between affected NRs in LRN group and unaffected NRs in controls at levels L1-L4 was considered statistically significant $(p<0.05)$. Receiver operating characteristic analysis revealed mean values to be $8 \mathrm{~mm}^{2}$ (CSA) for L1NR, $11.2 \mathrm{~mm}^{2}$ (CSA) for L2NR, $13.6 \mathrm{~mm} 2$ (CSA) for L3NR, and $17.8 \mathrm{~mm}^{2}$ (CSA) for L4NR. There was significant difference between $\triangle C S A$ of LRN patients and controls at L1 to L4 levels $(p<0.05)$. CSA measurements of lateral femoral cutaneous nerve $\left(8 \mathrm{~mm}^{2}\right)$ and femoral nerve $\left(58 \mathrm{~mm}^{2}\right)$ performed on high-resolution ultrasonography were significantly larger on the affected side as compared with the unaffected side.

Conclusion Radiculoplexus neuropathy of the lumbar plexus can be reliably diagnosed on high-resolution ultrasonography that can reveal nerve thickening. The laterality of affected NRs was significantly greater in LRN group when compared with controls. published online September 20, 2021
DOI https://doi.org/ 10.1055/s-0041-1735322. ISSN 0976-3147.

\footnotetext{
(C) 2021. Association for Helping Neurosurgical Sick People. All rights reserved.

This is an open access article published by Thieme under the terms of the Creative Commons Attribution-NonDerivative-NonCommercial-License, permitting copying and reproduction so long as the original work is given appropriate credit. Contents may not be used for commercial purposes, or adapted, remixed, transformed or built upon. (https://creativecommons.org/ licenses/by-nc-nd/4.0/)

Thieme Medical and Scientific Publishers Pvt. Ltd., A-12, 2nd Floor, Sector 2, Noida-201301 UP, India
} 


\section{Introduction}

Lumbar plexus comprises a large number of nerves, has a complex anatomy, and encompasses a broad spectrum of pathologies. "Lumbar radiculoplexus neuropathy" is a clinical syndrome that can occur with both motor and sensory disorders or a combination of both. The traditional diagnosis of lumbar radiculoplexus neuropathy (LRN) has been based on a classical sequence of symptoms and targeted electrodiagnostic examination by means of electromyography. ${ }^{1}$ Imaging modalities such as computed tomography and magnetic resonance imaging (MRI) were primarily used to compute space requirements and for planning biopsy. ${ }^{2}$ High diagnostic accuracy related to CSA enlargement of nerves indicating pinched nerve secondary to trauma and radiculopathy has been reported in recent ultrasonographic studies. ${ }^{3}$ Ultrasonography reliably indicates the level of lumbar radiculopathy by assessing edema mesial to the site of compression.

\section{Objective of the Study}

This case-control study investigated the factors leading to the diagnosis of LRN on high-resolution ultrasonography in diabetics.

\section{Materials and Methods}

\section{Patients}

This case-control study was undertaken at a tertiary care hospital between July 2017 and June 2019 on 15 patients with symptoms of LRN (IRB/IRC approval: IEC/SJH/31/2017). Fifteen healthy volunteers with no symptoms or clinical signs of LRN were included in the control group. A written informed consent was mandatorily obtained from patients in cases and control groups prior to their enrollment in the study. Diabetics who presented with radiculopathy, lower back pain with/without radiation to the lower extremities, numbness, paresthesias, weakness, aberrant lower limb deep tendon reflexes, or sensory changes were included in the study. MRI of the lumbosacral spine is necessary to exclude cases that disclose disc degeneration of the lumbar spine that causes compression of the lumbar NRs. Subjects who presented with trauma, myopathy, and collagen vascular diseases were excluded from the study.

\section{Ultrasonographic Measurements}

Ultrasonography was performed using a scanner with a highresolution 7.5 MHz linear array probe (Logiq 500 MD, GE Medical Systems, Wisconsin, United States). High-resolution ultrasonography examination was conducted on LRN patients' group and controls in lateral decubitus position. Examination was performed in brightness mode of the ultrasound machine with a linear array probe while standing behind the subject. On ultrasonography, the nerve roots (NRs) were identified on axial sections. The diameter and the transverse diameter of L1NR, L2NR, L3NR, and L4NR were computed, and cross-sectional area (CSAs) were ascertained at a point in the lateral recess located adjacent to the vertebral body. This case-control study assessed four components: (1) The CSAs of L1NR, L2NR, L3NR, and L4NR of diabetic patients were compared with controls. (2) The difference in laterality of CSA $\left(\Delta C S A \mathrm{~mm}^{2}\right)$ between LRN patients and controls was computed at L1NR, L2NR, L3NR, and L4NR nerve levels. (3) The difference attributed to CSAs between affected NRs in LRN group and unaffected NRs in controls was computed at levels L1 to L4. (4) The cutoff values, sensitivity, and specificity of the CSA and $\triangle$ CSA for L1NR, L2NR, L3NR, and L4NR were compared. On highresolution ultrasonography, femoral nerve was localized lateral to the femoral artery in the femoral triangle beneath the inguinal ligament. Measurements of bilateral femoral nerves at the level of L3 to L4 were calculated.

\section{Statistical Analysis}

Statistical analysis was performed using the Microsoft Excel 2013 (Microsoft Corp., Redmond, Washington, United States) and SPSS Statistical Package (version 20.0) (IBM SPSS Statistics for Windows, V.20.0, IBM Corp., Armonk, New York, United States). Descriptive statistics for categorical variables were reported using numbers and percentages. Normal distributions with regard to CSAs of L1NR, L2NR, L3NR, and L4NR in controls were tested using the Shapiro-Wilk test. Further, a nonparametric test such as Mann-Whitney U test was utilized for comparison of differences in laterality at L1, L2, L3, and L4 nerve levels. Wilcoxon signed-rank test was used for the comparison of CSAs at levels L1NR, L2NR, L3NR, and L4NR in LRN group. Cutoff values, sensitivity, and specificity of the CSA and $\triangle$ CSA for L1NR, L2NR, L3NR, and L4NR levels were calculated based on receiver operating characteristic (ROC) curve. The outcomes were demonstrated as interquartile range and median with $p$-values $<0.05$ being considered significant.

\section{Results}

Fifteen subjects ( 9 males and 6 females) with mean age of $52 \pm 12$ years (range: $30-80$ years) were included in the control group. In the LRN patients' group, 5 subjects were female and 10 subjects were male with a mean age of $48 \pm 10$ years (range: $32-78$ ). LRN for L1NR, L2NR, L3NR, and L4NR was diagnosed in 2, 3, 6, and 4 patients, respectively. There was no statistical significance attributed to age $(p=0.59)$. However, statistical significance was attributed to sex $(p=0.004)$ between LRN subjects and controls. In controls, the median CSAs were calculated as 7.4 (L1NR), 8.2 (L2NR), 8.8 (L3NR), and $9.8 \mathrm{~mm}^{2}$ (L4NR). In LRN subjects, the median CSAs of the affected side were 10.2 (L1NR), 12.8 (L2NR), 14.0 (L3NR), and $18.6 \mathrm{~mm}^{2}$ (L4NR) and were significantly higher as compared with those of unaffected side with values of 7.3 (L1NR), 8.3 (L2NR), 8.7 (L3NR), and $10.0 \mathrm{~mm}^{2}$ (L4NR). There was significant difference between median CSAs of L1NR, L2NR, L3NR, and L4NR in LRN patients' group as compared with controls $(p<0.05 ;-$ Table 1$)$. Additionally, the median CSAs of L1NR, L2NR, L3NR, and L4NR in patients with 
794 Lumbar Radiculoplexus Neuropathy in Diabetics Ravikanth

Table 1 Comparison of nerve root measurements in the LRN group versus the control group

\begin{tabular}{|l|l|l|l|l|l|}
\hline Cross-sectional area & L1NR & L2NR & \multicolumn{1}{l|}{ L3NR } & L4NR \\
\hline \multicolumn{2}{|l|}{} & 7.4 & 8.2 & 8.8 & 9.8 \\
\hline Control & $(6.1-8.4)$ & $(6.8-10.0)$ & $(7.4-10.6)$ & $(8.3-12.1)$ \\
\hline IQR & \multicolumn{5}{|l|}{} \\
\hline LNR group & 7.3 & 8.3 & 8.7 & 10.0 \\
\hline Unaffected side & $(6.3-8.7)$ & $(7.2-10.6)$ & $(7.7-11.0)$ & $(8.6-12.8)$ \\
\hline IQR & 10.2 & 12.8 & 14.0 & 18.6 \\
\hline Affected side & $(8.8-12.1)$ & $(10.7-14.4)$ & $(12.2-16.3)$ & $(16.4-20.1)$ \\
\hline IQR & 2 & 3 & 6 & 4 \\
\hline No. of patients & 0.62 & 0.54 & 0.42 & 0.63 \\
\hline$p$-Value (unaffected vs. control side) & $<0.05$ & $<0.05$ & $<0.05$ & $<0.05$ & $<0.05$ \\
\hline$p$-Value (affected vs. unaffected side) & $<0.05$ & $<0.05$ & $<0.05$ \\
\hline$p$-Value (affected vs. control side) & & & \\
\hline
\end{tabular}

Abbreviations: IQR, interquartile range; LRN, lumbar radiculoplexus neuropathy; NR, nerve root.

regard to the unaffected side demonstrated no statistical significance between LRN subjects and controls (L1: $p=0.62$, L2: $p=0.54$, L3: $p=0.42$, L4: $p=0.63 ;$ - Table 1) The median $\Delta$ CSAs were calculated as 1.8 (L1NR), 2.4 (L2NR), 3.1 (L3NR), and $4.1 \mathrm{~mm}^{2}$ (L4NR) in controls. Median $\triangle$ CSAs at L1 to L4 levels in LRN patients were calculated as 4.4, 6.3, 9.2, and $12.8 \mathrm{~mm}^{2}$. Significant statistical difference $(p<0.05)$ was noted in the $\triangle$ CSAs values of L1NR, L2NR, L3NR, and L4NR between the groups of LRN patients and controls (-Table 2 ). Threshold measurements related to $\triangle$ CSA and CSA were obtained using the ROC curve ( - Table 3 ). However, no statistical significance was attributed to $\triangle \mathrm{CSA}$ and CSA for L1NR or

Table 2 Laterality comparison in the LRN group versus the control group

\begin{tabular}{|c|c|c|c|c|}
\hline \multicolumn{5}{|c|}{ Laterality of the cross-sectional area $(\Delta \mathrm{CSA})$} \\
\hline & L1NR & L2NR & L3NR & L4NR \\
\hline Control & 1.8 & 2.4 & 3.1 & 4.1 \\
\hline IQR & $(0.7-2.4)$ & $(1.1-3.8)$ & $(1.3-4.1)$ & $(1.8-5.5)$ \\
\hline LRN group & 4.4 & 6.3 & 9.2 & 12.8 \\
\hline IQR & $(2.8-6.5)$ & $(4.4-8.9)$ & $(6.3-12.2)$ & $(10.1-14.7)$ \\
\hline No. of patients & 2 & 3 & 6 & 4 \\
\hline$p$-Value & $<0.05$ & $<0.05$ & $<0.05$ & $<0.05$ \\
\hline
\end{tabular}

Abbreviations: IQR, interquartile range; LRN, lumbar radiculoplexus neuropathy; NR, nerve root.

Table 3 Diagnostic accuracy of high-resolution ultrasonography in the diagnosis of lumbar radiculoplexus neuropathy

\begin{tabular}{|l|l|l|l|l|l|}
\hline Threshold & Sensitivity (\%) & Specificity (\%) & Threshold value & AUC (95\% CI) & $p$-Value \\
\hline L1NR-CSA & 88 & 92 & 9.8 & $0.95(0.94-0.96)$ & 0.44 \\
\hline L1NR- $\Delta$ CSA & 89 & 94 & 3.8 & $0.90(0.89-0.91)$ & \\
\hline L2NR-CSA & 91 & 92 & 11.2 & $0.94(0.93-0.95)$ & 0.67 \\
\hline L2NR- $\Delta$ CSA & 78 & 96 & 5.1 & $0.89(0.88-0.90)$ & \\
\hline L3NR-CSA & 92 & 98 & 13.6 & $0.96(0.95-0.97)$ & $<0.05$ \\
\hline L3NR- $\Delta$ CSA & 84 & 96 & 7.8 & $0.91(0.90-0.92)$ & $<$ \\
\hline L4NR-CSA & 90 & 94 & 17.8 & $0.97(0.96-0.99)$ & $<0.05$ \\
\hline L4NR- $\Delta$ CSA & 92 & 98 & 8.8 & $0.93(0.91-0.95)$ & \\
\hline
\end{tabular}

Abbreviations: AUC, area under the curve; $\mathrm{Cl}$, confidence interval; CSA, cross-sectional area; LRN, lumbar radiculoplexus neuropathy; NR, nerve root. 
L2NR ( $p=0.44$ and 0.67). CSA measurements of lateral femoral cutaneous nerve $\left(8 \mathrm{~mm}^{2}\right)$ and femoral nerve $\left(58 \mathrm{~mm}^{2}\right)$ performed on high-resolution ultrasonography were significantly larger on the affected side as compared with the unaffected side.

\section{Discussion}

Diabetic lumbosacral radiculoplexus neuropathy (DLSRPN) begins as focal pain as the presenting initial symptom, which gradually progresses to generalized and bilateral weakness. In diabetics, quantitative testing revealed the presence of sensory and autonomic neuropathy. Although weakness related to radiculoplexus neuropathy has been described by diabetic patients as the most disabling symptom, the mechanism causing DLSRPN has still been a subject of debate. ${ }^{4}$ Hyperglycemia and ischemic damage have been proposed as primary mechanisms causing DLSRPN. ${ }^{5}$ DLSRPN is a unique entity and can be clearly distinguished from distal diabetic sensorimotor neuropathy, which is more common compared with DLSRPN with an insidious onset, symmetric, and symptoms are time dependent, affecting only the sensory functions. The proposed mechanism as the cause of DLSRPN is attributed to abnormal glucose metabolism causing diabetic distal sensorimotor neuropathy and microvasculitis. Various terms have been used to describe DLSRPN that include diabetic mononeuritis multiplex, diabetic polyradiculopathy, diabetic amyotrophy, and Bruns-Garland syndrome. ${ }^{6}$ DLSRPN is a monophasic illness that has symptoms worsening for $\sim 6$ months and then gradually shows improvement. A variant form of diabetic lumbosacral plexopathy has been described that presents as painless motor predominant neuropathy in the lower limb. Patients with this form of diabetic lumbosacral plexopathy present with a greater degree of weakness and symptoms progress insidiously and symmetrically than the typical painful DLSRPN. Histopathological examination of nerves in diabetes-related neuropathy demonstrated ischemiarelated microvasculitis to be the cause and not inflammation-related demyelination as the underlying mechanism. ${ }^{7}$ Expansion at both the proximal and distal ends of NR caused due to entrapped peripheral NRs was reported by Simon et al. ${ }^{8}$ The current study also determined that increased CSAs of lumbosacral NRs mesial to the site of compression were found in LRN subjects. External compression of a peripheral nerve in entrapment disease causing internal ultrastructural changes due to elevated venous perfusion, leading to augmented pressures within the NRs with resultant edema causing engorgement, was demonstrated by Bianchi et al. ${ }^{9}$ A study by Rao ${ }^{10}$ reported that in entrapment neuropathies, increased vascular permeability exhibited by intrinsic blood vessels may cause fibrosis in affected NRs. No literature was available on the web precisely comparing CSA or $\triangle \mathrm{CSA}$ between control subjects and diabetic LRN patients.

\section{Limitations of the Study}

The limitation of this study includes relatively small number of cases and only diabetic LRN patients were considered for the study. External validity may be limited in the current study due to single-center setup.

\section{Conclusion}

In conclusion, high diagnostic accuracy of CSA and $\triangle$ CSA thresholds was identified in diabetic LRN patients on ultrasonography. Radiculoplexus neuropathy of the lumbar plexus can be reliably diagnosed on high-resolution ultrasonography that can reveal nerve thickening. The laterality of affected NRs was significantly greater in LRN group when compared with controls.

\author{
Funding \\ None. \\ Conflict of Interest \\ None declared.
}

\section{References}

1 Dyck PJ, Windebank AJ. Diabetic and nondiabetic lumbosacral radiculoplexus neuropathies: new insights into pathophysiology and treatment. Muscle Nerve 2002;25(04):477-491

2 Taylor BV, Kimmel DW, Krecke KN, Cascino TL. Magnetic resonance imaging in cancer-related lumbosacral plexopathy. Mayo Clin Proc 1997;72(09):823-829

3 Singh Y, Dixit R, Singh S, Garg S, Chowdhury N. High resolution ultrasonography of peripheral nerves in diabetic peripheral neuropathy. Neurol India 2019;67(Supplement):S71-S76

4 Garces-Sanchez M, Laughlin RS, Dyck PJ, Engelstad JK, Norell JE, Dyck PJ. Painless diabetic motor neuropathy: a variant of diabetic lumbosacral radiculoplexus Neuropathy? Ann Neurol 2011;69(06):1043-1054

5 Sinnreich M, Taylor BV, Dyck PJ. Diabetic neuropathies. Classification, clinical features, and pathophysiological basis. Neurologist 2005;11(02):63-79

6 Dyck PJ, Norell JE, Dyck PJ. Non-diabetic lumbosacral radiculoplexus neuropathy: natural history, outcome and comparison with the diabetic variety. Brain 2001;124(Pt 6):1197-1207

7 Kawamura N, Dyck PJ, Schmeichel AM, Engelstad JK, Low PA, Dyck PJ. Inflammatory mediators in diabetic and non-diabetic lumbosacral radiculoplexus neuropathy. Acta Neuropathol 2008;115(02):231-239

8 Simon NG, Ralph JW, Poncelet AN, Engstrom JW, Chin C, Kliot M. A comparison of ultrasonographic and electrophysiologic 'inching' in ulnar neuropathy at the elbow. Clin Neurophysiol 2015;126(02):391-398

9 Bianchi S, Montet X, Martinoli C, Bonvin F, Fasel J. High-resolution sonography of compressive neuropathies of the wrist. J Clin Ultrasound 2004;32(09):451-461

10 Rao R. Neck pain, cervical radiculopathy, and cervical myelopathy: pathophysiology, natural history, and clinical evaluation. Instr Course Lect 2003;52:479-488 